\title{
LC-MS/MS validation for drug of abuse testing utilizing a split sample oral fluid collection system.
}

1 Marissa Howard ${ }^{1}$, Paul Russo ${ }^{1}$, Amanda Haymond ${ }^{1}$, Valerie Cruz Ortiz $^{1}$, Sydney R. Andes ${ }^{1}$,

2 Virginia Espina ${ }^{1}$, Alessandra Luchini ${ }^{1}$, Lance A. Liotta*1, and Raouf Guirguis ${ }^{2}$

$3{ }^{1}$ Center for Applied Proteomics and Molecular Medicine, George Mason University, Manassas, VA, 4 USA.

$5{ }^{2}$ Affiliate Faculty, College of Systems Biology, College of Science, George Mason University,

6 Manassas, VA, USA.

7 * Correspondence: lliotta@gmu.edu

8 Keywords: oral fluid, drugs of abuse, mass spectrometry, point of care testing, split sample, 


\section{Abstract}

11

12
The Substance Abuse and Mental Health Services Administration (SAMHSA) recently authorized oral fluid (OF) as a preferable biofluid for drugs of abuse (DOA) screening compared to urine, and they required that each screening method be confirmed by a laboratory test. We developed a DOA mass spectrometry (MS) assay optimized for undiluted OF as a matching confirmatory test for the EZ-Saliva point of care (POC), split sample, rapid visual test. Using a double isotope ratio standardization, we achieved a limit of detection of $<0.3 \mathrm{ng} / \mathrm{mL}$ for seven DOAs, with high precision in undiluted patient $\mathrm{OF}(\mathrm{CV}<7.2 \%)$, linearity of $\mathrm{R}^{2}=0.99$, lack of interference $(<1.0 \%)$ by a panel of interfering compounds at 1000-fold excess, and a dynamic range of 0-850 ng/mL, from a consented population of $\mathrm{N}=84$ self-reported THC users using the collection device (device yield $>90 \%$ ). Stability from degradation exceeded 72 hours. The lateral flow immunoassay strips of the POC exhibited a dose-dependent response, with a $90 \%$ sensitivity and $100 \%$ specificity for $\mathrm{N}=22$ selfreported, THC patient OF, digitized for quantitation. We conclude that the split sample POC device in combination with the MS assay meets the SAMHSA stated requirements for a POC test with a laboratory confirmation. Split sample collection has significant advantages because it minimizes potential error created by taking a separate OF sample for laboratory confirmation. We recommend scaling to a larger validation study set and quantification of user OF THC levels that correlate with driver impairment levels. 
31 Recreational marijuana use is legal in 11 USA states as well as the District of Columbia. ${ }^{12}$ In 2018 , the drug, with $52 \%$ of people surveyed between the ages of 18-26 reporting the use of cannabis during their lifetime, and $35 \%$ reporting cannabis use in the month prior to the survey. ${ }^{1-2}$ After cannabis, synthetic opioids are the second-most consumed drugs of abuse in North America. Roughly $4.1 \%$ of the US population (5.8\% of males, $2.5 \%$ of women) report driving while under the influence of illicit drugs of abuse, resulting in car accidents with a higher driver fatality rate than in accidents involving drivers under the influence of alcohol (44\% versus $38 \%$ in 2016). ${ }^{7-8}$ Additional health consequences associated with drug use include mental health disorders, viral infections, including HIV and Hepatitis $\mathrm{C}$, and liver cancer. ${ }^{24}$ These serious consequences of drug use lead to high societal costs estimated at 55.7 billion USD in 2007. Workplace earnings lost due to DOA use are estimated at 11.2 billion USD and police/criminal correctional units costs to enforce DOA misuse are 3.8 billion USD in $2007 .{ }^{5}$ However, despite the risk of marijuana use impairing the driving capabilities of the user, there currently exists no standardized oral fluid test for law enforcement to confirm drug use for suspected impairment. The Substance Abuse and Mental Health Services Administration (SAMHSA) regulates the required guidelines for testing patients or individuals suspected to be under the influence of drugs in the workplace, driving vehicles, or medical environments. In the past, most testing for drug of abuse (DOA) have used urine-based testing. Urine testing has inherent drawbacks for onsite testing and requires preservation methods (i.e. refrigeration) for additional confirmatory testing. In October of 2019, SAMHSA addressed the drawbacks of urine testing and authorized the use of oral fluid as a preferable biofluid for DOA testing. ${ }^{11}$ Compared to urine-based testing, DOAs within OF do not require renal clearance and are indicative of recent drug use through direct exposure by smoking and/or oral administration. ${ }^{6,10,13}$ In addition to recommending OF as a preferred matrix, SAMHSA requires all DOA screening tests to undergo an additional laboratory test confirming the primary screening result. Thus, in the DOA surveillance testing environment there is an unmet need for a rapid OF screening method matched to a highly sensitive confirmatory method for DOA measurement in OF.

To achieve this important need, two technical components need to be addressed. The first is a technology or tool for OF collection, rapid diagnosis, and storage for confirmatory test. This device must be tamper-proof to protect the OF/saliva sample chain of custody. Secondly, a robust, precise, accurate, and quantitative laboratory analysis method for undiluted OF samples needs to be matched and available in tandem to validate the screen results from the point-of-care device.

In the present study we introduce an OF mass spectrometry multiple reaction monitoring (MS/MRM) protocol for DOA detection that uses a combination of heavy and light isotope standards as internal calibrators. Secondly, we introduce a split sample point of care device, the EZ-Split Saliva II (ESS), that takes the same input of OF and splits the sample into a) a 5 DOA panel rapid lateral flow immunoassay (LFI), and, b) simultaneously securely aliquots the same OF for confirmatory analysis. We used the MS/MRM quantification method to evaluate the sensitivity, yield, linearity, and stability of 7 DOAs. Using this method we examined a cohort of self-reported THC positive OF samples to determine the distribution of THC levels in a given population and correlate THC patient data to the point of care (POC) diagnosis. The overall purpose of this study was to evaluate the split sample collection device for on-the-spot screening followed by liquid chromatography with tandem mass spectrometry (LC/MS-MS) confirmatory testing. 


\subsection{Reagents and Standard Solutions}

79

80

81

82

83

84

85

86

87

88

89

90

91

92

93

Optima LC/MS grade methanol (MeOH), LC/MS grade $0.1 \%$ formic acid in water, LC/MS grade formic acid ampules, optima grade dichloromethane (DCM), optima LC/MS grade isopropanol (IPA), hydrochloric acid $\mathrm{HCl}$, tert-butyl methyl ether, (MTBE), and hexane were purchased from Fisher Scientific. Ammonium hydroxide $\left(\mathrm{NH}_{4} \mathrm{OH}\right.$ was purchased from Sigma. DOA analytical standards: (+/-) amphetamine, (+/-) methamphetamine, cocaine, benzoylecgonine, morphine, phencyclidine, (-) delta9-THC, (+/-) amphetamine - D5, (+/-) methamphetamine - D5, cocaine - D3, benzoylecgonine D3, morphine - D3, phencyclidine - D5, and (-) delta9-THC - D3 were obtained as 1mg/mL solutions from Cerilliant (Round Rock, TX, USA). Interfering substances: Diphenhydramine HCl, Alprazolam, Dihydrocodeine, S-Nicotine, Caffeine, Cortisol, - Cotinine, Dextromethorphan, Ibuprofen, Naproxen, Prednisone, R-Pseudoephedrine, Amobarbital, Propranolol, Nicotinamide, Carbamazepine, Clobazam, Clonazepam, Valproic Acid, Verapamil, and Sertraline were purchased from Cerilliant (Round Rock, TX, USA). Stabilization buffer was purchased from Immunalysis (Pomona, CA). The Raptor Bipheyl LC column; 2.7um, 100mm x 2.1mm was purchased from Restek (Bellefonte, PA). Isolute SLE+ columns were purchased from Biotage (Charlotte, NC). The PRESSURE+ 48 positive pressure manifold was obtained from Biotage (Charlotte, NC).

\subsection{Oral fluid collection and human subjects.}

Saliva was collected from volunteers using mLife EZ Saliva-II Saliva (ESS) Based Drug Test kits (https://www.mlifedx.com/). The ESS device collects the OF in a sponge which splits the OF into a) a channel which communicates the OF to a lateral flow immunoassay DOA line, and, b) into a secure aliquot for later confirmatory mass spectrometry testing. The collected oral fluid is undiluted. The ESS displays a color change in the stem when the proper adequate volume of OF is collected.

All participants signed a written informed consent. The study received approval from the George Mason University Institutional Review Board. Saliva was self-collected by the participants under the direct observation of a study team member. Saliva was collected until the color indicator on the collection device turned red/pink. The wand was inserted into the test kit, and the test kit was placed in a Ziplock, plastic biohazard specimen transport bag, and stored at -20C prior to mass spectrometric analysis of THC.

Four sets of volunteers were analyzed. Set 1 contained N=84 patients whose OF was collected after self-reported marijuana recreational use in a group setting. This set was used to establish the expected dynamic range distribution of OF THC within an active-intake population. Set 2 contained $\mathrm{N}=39$ negative controls who reported no use of marijuana. This set was used to ascertain mass spectrometry analysis specificity. Set 3 contained $\mathrm{N}=21$ patients whose $\mathrm{OF}$ was collected after self-reported marijuana use. This set was analyzed in a blinded fashion and was used to determine performance of the LFIs in comparison with mass spectrometry analysis. 14/21 volunteer samples had adequate amount of OF in the collection device to be subjected to both MRM and LFI analysis. Set 4 was a pilot examination of the time course of THC concentration in OF and perceived drug impairment in one participant who smoked $0.5 \mathrm{~g}$ of medical marijuana. The last known use of marijuana compounds was $>30$ days prior to this event. Seven minutes post marijuana use, OF was self-collected by the participant using the mLife EZ Saliva-II Saliva Based Drug Test. OF was self-collected hourly for 3 hours, for a total of 4 specimens: 7 minutes, 1 hour, 2 hours, and 3 hours post marijuana use. Participant height and weight were 5' 9', and $200 \mathrm{lbs}$, respectively. 
121 Two hundred (200) $\mu \mathrm{L}$ of each undiluted OF sample was transferred to a low binding $1.5 \mathrm{~mL}$ centrifuge

122 tube; $600 \mu \mathrm{L}$ of stabilization buffer and $3 \mu \mathrm{L}$ of concentrated ammonium hydroxide were added, and all 123 samples vortexed.

124 The samples organic fraction, containing the DOA, was separated from the aqueous fraction using 125 supported liquid extraction columns. In order to do so, $800 \mu \mathrm{L}$ of stabilized OF sample was transferred 126 to $1 \mathrm{~mL}$ Biotage SLE+ Isolute columns, which were positioned on a Biotage PRESSURE+ 48 positive pressure manifold. After 5-minutes, elution from the columns was accomplished by the addition of $2.5 \mathrm{~mL}$ of DCM:IPA (95:5), followed by another addition of $2.5 \mathrm{~mL}$ of DCM:IPA (95:5). Each elution step was performed with a small pressure of about $0.5 \mathrm{psi}$, with a 5-minute waiting period between elution steps. Both elutions were collected into $12 \times 75 \mathrm{~mm}$ borosilicate glass test tubes. After the final elution, a higher pressure of about 10psi was used to empty the columns of all liquid into the glass tubes. To each glass tube, $100 \mu \mathrm{L}$ of $50 \mathrm{mM} \mathrm{HCl}$ in $\mathrm{MeOH}$ was added. Each tube was dried on a MicroVap at $40^{\circ} \mathrm{C}$ under about 4LPM nitrogen. Each sample was reconstituted with $200 \mu \mathrm{L}$ of $0.1 \%$ formic acid in $\mathrm{MeOH}$. Fifty (50) $\mu \mathrm{L}$ of each test tube was transferred to an autosampler vial for MRM analysis.

\subsection{DOA internal standard and calibration curves.}

137 DOA heavy isotopes (DOA-Hv, (+/-) amphetamine, (+/-) methamphetamine-D5, cocaine-D3, 138 benzoylecgonine-D3, morphine-D3, phencyclidine-D5, and (-) delta9-THC-D3) were used as internal standards for DOA quantification, and spiked in OF samples at a concentration of $50 \mathrm{ng} / \mathrm{mL}$. Linearity of the assay was assessed by regression of standard curves expressed as light/heavy isotope ratio. For all DOAs, light isotopes were spiked in OF at the following concentrations: $100 \mathrm{ng} / \mathrm{mL}, 50 \mathrm{ng} / \mathrm{mL}$, $25 \mathrm{ng} / \mathrm{mL}, 12.5 \mathrm{ng} / \mathrm{mL}, 6.25 \mathrm{ng} / \mathrm{mL}, 3.125 \mathrm{ng} / \mathrm{mL}$, and $1.56 \mathrm{ng} / \mathrm{mL}$.

\subsection{Mass Spectrometry Analysis}

144 LC-MRM experiments were performed on a TSQ Quantum XL triple quadrupole mass spectrometer (ThermoFisher) equipped with an Accela HPLC and autosampler system (ThermoFisher). Drugs of abuse were separated using a Raptor BiPhenyl, 100mm x $2.1 \mathrm{~mm}, 2.7 \mu \mathrm{m}$ LC column (Restek). The mobile phase consisted of $0.1 \%$ aqueous formic acid (mobile phase A) and $0.1 \%$ formic acid in methanol (mobile phase B). After injection, the DOA were eluted using a gradient of 5\% - 40\% B in 2 minutes, $40 \%-60 \% \mathrm{~B}$ in 5 minutes, $60 \%-90 \% \mathrm{~B}$ in 4 minutes, and finally back to $5 \% \mathrm{~B}$ over 2 minutes. Flow rate was set at $300 \mu \mathrm{L} / \mathrm{min}$. Spray voltage was set at $+4000 \mathrm{~V}$ and nitrogen sheath gas pressure was set at 40. The first quadrupole was operated at $0.7 \mathrm{amu}$ (FWHM), and set to pass 14 different precursor $\mathrm{m} / \mathrm{z}$. The second quadrupole was filled with $1.5 \mathrm{mT}$ Torr of argon gas for fragmentation. The third quadrupole was set to cycle through 42 different transitions ( 3 transitions per precursor). The cycle time was set to 1 second, which equates to a dwell time of $24 \mathrm{msec}$. All optimal precursor, transition, collision energy, and tube lens values were determined by direct infusion of all drugs of abuse in $\mathrm{MeOH}$ into the mass spectrometer prior to conducting any LC-MRM analyses. These values are all shown in Table S2. All MRM data was imported into, and analyzed with, Skyline v 4.1 (University of Washington, Seattle WA, Michael MacCoss laboratory). All procedures followed guidelines under CAP/CLIA certification in the authors' high complexity CAP CLIA clinical laboratory.

\subsection{Interfering Substances}


162 In order to test assay specificity, the interfering substances listed in Supplementary Table 1 were diluted

163 in methanol and added to OF samples containing DOAs in the following amounts:

1641 1. $100 \mathrm{ng} / \mathrm{mL} 7 \mathrm{DOA}+100 \mathrm{ng} / \mathrm{mL}$ each for all 22 interfering substances in a unique sample.

165 2. $100 \mathrm{ng} / \mathrm{mL} 7 \mathrm{DOA}+1000 \mathrm{ng} / \mathrm{mL}$ each for all 22 interfering substances in a unique sample .

$166 \quad 3 . \quad 100 \mathrm{ng} / \mathrm{mL} 7 \mathrm{DOA}$ only

167 4. $100 \mathrm{ng} / \mathrm{mL}$ each for all 22 interfering substances in a unique sample

\subsection{EZ-Saliva Kit DOA stability assessments at 24 hours, 72 hours, and 1 week.}

169 All seven DOAs were spiked in negative donor OF at a concentration of $50 \mathrm{ng} / \mathrm{L}$. $1 \mathrm{~mL}$ of DOA spiked 170 OF were placed into EZ-Saliva II tamperproof mass spectrometry collection vials. The vials were 171 capped, placed in a foil bag out of the light, and left at room temperature. After 24 hours, 72 hours, and 1721 week, $200 \mu \mathrm{L}$ of saliva from each sample was transferred to $1.5 \mathrm{~mL}$ low binding centrifuge tubes. 173 Each time saliva was transferred, the vial was recapped, and placed back into the foil bag and left at 174 room temperature. Samples were subjected to mass spectrometry as described above.

\section{$175 \quad 2.8 \quad$ Lateral Flow Immunoassay}

176 EZ-Saliva Lateral Flow Immunoassay Strips were donated by MLife Diagnostics. The 5-panel LFI 177 strips were for the following DOAs: Amphetamine, Methamphetamine, Cocaine, THC, and Opiates. 178 The cut-offs for the LFIs were indicated on the package insert. A dose response curve $(0-100 \mathrm{ng} / \mathrm{mL})$ 179 of the DOAs (excluding THC), were spiked into a neat, undiluted oral fluid sample. The 5-panel 180 wicking strips were submerged into the spiked-OF sample and held at a $90^{\circ}$ angle for 5 minutes. After 181 the sample completely flowed to the absorbent pad, an image was taken of the LFI panel. Digital image 182 was acquired using an iPhone 6S. Densitometry analysis was performed on the test and control lines 183 of the LFI using ImageJ software. All experimentation was performed using disposable glassware.

\section{$184 \quad 2.9$ Statistical Methods}

185 Linear regression of light/heavy isotope peak area ratio versus standard concentration was used to 186 build calibrator curves. Variation of DOA concentrations in different experimental conditions was 187 assessed by t test. Discriminatory performance of THC LFI strips compared to binary outcome of 188 MRM analysis was assessed via received operating characteristics (ROC) analysis. All statistical analyses were performed using GraphPad Prism Version 8.0 Software. 


\subsection{Rapid Point of Care Oral Fluid Collection System allows for dual simultaneous diagnosis and storage for downstream confirmatory analysis.}

Driving after use of illicit drugs increases a person's risk of being seriously injured or killed in a driving accident up to three-fold. ${ }^{6,7}$ Compared to subjective officer-based assessments of impaired driving, a POC rapid diagnostic screening device, at the roadside, can contribute objective information to inform the officer's judgement. Oral fluid DOA levels may reflect the current or recent use of DOA and can be easily collected in a public setting under observation. OF has been documented to reflect DOA systemic levels within 1 hour after intake. ${ }^{4}$ In contrast, urine collection must be done in private, and the urine levels may lag 24 hours behind the peak level of DOA, and its associated impairment. The EZ-Split Saliva II (ESS) device is a novel OF collection system for multiplexed diagnosis and secure storage of sample (Figure 1). ${ }^{21-22}$ The device collects the saliva in a sponge which splits the OF into a) a channel which communicates the OF to a LFI DOA line, and, b) into a secure aliquot for later confirmatory testing. The ESS LFIs utilize a competitive immunoinhibitory method opposite to the double-antibody sandwich immune assay. Specifically, the immunoinhibitory lateral flow assay has a pre-bound analyte antigen on the test line. When the patient's analyte binds to the labeled monoclonal antibody, this prevents the labeled antibody from binding to the bound antigen on the test line. This type of immunoassay is necessary for DOA screening, compared to a sandwich immunoassay because a small molecular weight drug does not present the available dual non-competing epitopes required for a typical sandwich assay. A major advantage the ESS device compared to single aliquot POC OF tests is that the device collects, in parallel, the same OF confirmatory sample, approximately $1.9 \mathrm{~mL}$ to $2.5 \mathrm{~mL}$, into a secure, tamper proof collection vial. ${ }^{4,16}$ The ESS device collects undiluted saliva for LFI analysis and confirmation. In contrast, commercialized OF DOA screening devices such as Quantisil and the Draeger DDT5000 require an OF buffer dilution step that can reduce sensitivity. Depending on the collection yield, swab protocol, and mouth residence time an individual OF sample may not collect sufficient volume for further confirmatory testing within the same device kit. ${ }^{6,12,18,20}$ To address this source of preanalytical variability, the ESS displays a color change in the stem to insure that the proper adequate volume of OF is collected. Laboratory confirmation testing of suspected DOA samples is an essential step to legally verify if an individual was exceeding a legal limit of OF DOA levels at that moment in time. Due to the variability of OF collection and storage methods, it is impractical for a technician or officer to take more than one OF sample for diagnosis and confirmation testing respectively. Furthermore, taking multiple OF samples increases the variability of time-dependent DOA concentration within the OF leading to poorer quantitation and inaccuracies. The split sample system introduced here overcomes these difficulties with dual OF collection sponges (Figure 1). The ESS collection vial ensures that the same neat, undiluted OF sample can be utilized for downstream confirmatory analysis (LC/MS-MS). This tamper proof vial meets chain of custody requirements and ultimately, protects the patients by removing the subjectivity and uncertainty regarding the LFI diagnosis. Overall, the ESS kit has features supporting value as a screening in the workforce or during police traffic stops.

\subsection{LC/MS-MS Isotope ratio analysis is highly specific and precise.}

Previous published MS protocols for OF DOA testing report that precision and linearity of the assay is affected by analytical sensitivity, variance, and linearity, volume of OF, OF dilution by stabilization buffers, and OF matrix effects. ${ }^{17,19}$ Herein we introduce a simple MS MRM OF DOA assay that utilizes the neat, undilute OF collected in the tamper proof vial of the ESS kit. We follow guidelines under CAP/CLIA certification in the authors' high complexity CAP CLIA Clinical Lab. The MS MRM 
protocol is user-friendly and rapid (Figure 2A). Our protocol introduces heavy isotope reference standards for DOA quantitation. The panel of DOAs included morphine, amphetamine (AMP), methamphetamine, Cocaine (COC), benzoylecgonine (BZE), phencyclidine (PCP), and Delta-9tetrahydrocannabinol (THC) (Figure 2 B). ${ }^{14,15}$ Assurance of accuracy and linearity, particularly around the legal limit cut point is critically important because of the legal implications. For all DOAs, the assay was linear over the range of $1.56 \mathrm{ng} / \mathrm{mL}$ to $100 \mathrm{ng} / \mathrm{mL}$ (Figure 2 C). In order to assess ion suppressive matrix effects, DOAs were spiked into $\mathrm{N}=5$ DOA-free $\mathrm{OF}$ samples at different concentrations. Matrix effects were evident when considering DOA peak areas without the addition of heavy isotope standardization (AMP CV=40.33\% across 5 volunteer samples at $100 \mathrm{ng} / \mathrm{mL}$ ) (Figure 2 D). Importantly, when heavy isotope standard were used, DOA light/heavy isotope ratio were much less susceptible to patient-to-patient variability and showed improved linearity (R>0.99) (AMP CV= $6.48 \%$ at $100 \mathrm{ng} / \mathrm{mL}$ ) (Figure 2 D-E, Supplemental 1 A-L).

\subsection{High precision of the LC/MS-MS method of DOA identification from OF has no significant measurable interference from potential OF contaminants.}

To further analyze the LC-MS/MS precision we spiked in $50 \mathrm{ng} / \mathrm{mL}$ each of $7 \mathrm{DOA}$ into $\mathrm{N}=5$ DOAstandard/heavy isotope ratio quantitation, the average DOA concentration calculated was $50.0 \mathrm{ng} / \mathrm{mL}$, demonstrating excellent agreement with the concentration of the DOA spiked into the OF. Individual quantification of each DOA within the OF led to less than $10 \% \mathrm{CV}$ values for each drug tested (Figure 3 A,B). Furthermore, the LC-MS/MS limits of detection for each DOA are below $0.3 \mathrm{ng} / \mathrm{mL}$, well below the legal cut-off limits (Figure $3 \mathbf{C}$ ). To validate the specificity of the LC/MS-MS detection method against potential OF interfering substances, we spiked in $100 \mathrm{ng} / \mathrm{mL}$ and $1000 \mathrm{ng} / \mathrm{mL}$ of 22 different commonly prescribed medications (Supplemental Table 1) along with 100ng/mL of a 7 DOA mix into DOA-free donor OF sample. The peak areas of the individual DOA spectra were not substantially affected by either concentration of the 22 interfering substances (Figure 3 D). Lower peak area values for specific DOA tested can be attributed to drug specific degradation rates. A specific example is shown in Figure 3E for Amphetamine, where even at a level of $1000 \mathrm{ng} / \mathrm{mL}$ of the 22 interfering substances (vs 100ng/mL of the 7 DOA), all 3 transition ions, including the ion used for quantification $(\mathrm{m} / \mathrm{z}$ 91.045) are unaffected. The insert demonstrates that the 22 interfering substances at $1000 \mathrm{ng} / \mathrm{mL}$ alone (no DOA) show nothing but background when detecting the 3 transition ions for Amphetamine. Overall, the methodology developed shows strong specificity to the DOA of interest with no disturbance from high concentrations of non-DOA OF interfering substances.

\subsection{Split Saliva Collection Device successfully recovers and prevents DOA degradation for over} 72 hours.

We tested the collection efficiency of the ESS devices $(\mathrm{N}=6)$ wicking sponge pads by spiking in 50 $\mathrm{ng} / \mathrm{mL}$ of a 6 DOA (morphine, AMP, methamphetamine, COC, BZE, and Delta-9tetrahydrocannabinol (THC)) mix into DOA free OF. The spiked OF was collected by the ESS sponge pads and then deposited into the test cassette. Samples were analyzed using the MS method above. The percentage of DOA recovery from the wicking sponge pad for each sample ranged from 85-99\% which further demonstrates the ESS POC device having a high yield of recovery. Cocaine had the lowest recovery rate of the drugs tested and delta-9-THC had the most variable rate of recovery from the sponge pad (Figure 4 A). Next the stability of the glass vial was tested by spiking in $50 \mathrm{ng} / \mathrm{mL}$ of a DOA mix into $\mathrm{N}=3$ unique donated DOA free OF. The sample was processed through the collection kit and stored in the opaque, sealed package in which the kit arrives in. At 24h, 72h, and 1 week time points the collection vial was analyzed by MRM in triplicate. We found that a majority of the DOAs tested remain at $50 \mathrm{ng} / \mathrm{mL}$ at 1 week time, however, cocaine rapidly metabolizes into benzoylecgonine (BZE), cocaine's primary metabolite, post-24h (Figure 4 B). ${ }^{9}$ BZE's concentration increases inversely 
to cocaine's metabolization at the $48 \mathrm{~h}$ and 1 week time points. This present data indicates that MS quantified concentrations of DOAs within unbuffered OF is acceptably stable for 72 hours at room temperature or 4 degrees $\mathrm{C}$. This timeline fits directly within the standard shipping timelines for police officers to send samples to confirmatory labs. Our data indicates that holding the OF sample more than 72 hours, may be associated with a breakdown of certain DOAs into their respective metabolic side forms that are different from the in vivo native state of the drug. Specifically, for cocaine OF confirmation testing, we recommend the total level of cocaine and BZE be calculated concurrently, with their values combined, in order to determine the most accurate levels at the time of collection. These data support the need for further pharmacodynamic time course studies of emerging DOA tests employing OF samples, recognizing the potential for metabolic conversion in vivo and ex vivo under common storage conditions to provide the most accurate measurement of the drug.

\subsection{Competitive LFIs within the Collection Device demonstrate a dose-dependent response for non-THC DOAs.}

The LFIs within the ESS device employ a competitive immunochromatography method where the colloidal gold bead is labelled with the antibody against the DOA antigen. When the antigen is present (positive sample) and binds to the antibody labelled bead, the bead migrates past the test line. However, when the antigen is not present (negative sample), the antibody labelled bead arrests at the test line which contains the antigen bound to an antibody. Therefore, a negative sample presents with two lines, whereas a positive samples presents with only one line, the top control line (Figure 5 A). Competitive immunoassays are required for small molecule LFI assays because small drug analyte molecules lack the space for dual binding epitopes that are required for sandwich-based immunoassays. We tested and quantified the LFI response to a dose response of 4 DOAs. Densitometry analysis using Image $\mathbf{J}$ software quantified the pixel intensity of the test and control lines. The DOAs, excluding THC, show a clear dose dependent response on the LFI test line (Figure 5 B). Native THC was extremely absorptive to polymeric surfaces of the ESS collection device, whereby the antigen failed to reach the LFI test line resulting in a false negative diagnosis (Figure $5 \mathbf{C}$ ). We will mitigate this drawback using chemical additives that increase THC solubility and minimize loss. Despite the value of visual rapid screening, these tests can suffer from the subjectivity of the visual reader and the lighting conditions. Additionally, since the mechanics of the LFI are opposite to the conventional sandwich based LFIs, inexperienced or distracted users may not read the test properly. Digital scanning was effective in measuring and identifying a dose response curve for the DOAs tested. We recommend that the all DOA LFI based rapid screening tests incorporate a low cost digital based quantification of the test line in order to reduce the subjectivity of the user, and protect the individual who is being tested.

\subsection{MS Method Accurately Quantifies the Distribution of THC within a Population of Self- Reported THC Positive OF.}

Using the ESS kit, $\mathrm{N}=84$ volunteers donated OF after self-reported cannabis use (smoking) in a group setting. All samples were collected within the same time period. The goal was to evaluate the expected required dynamic range for THC oral fluid levels for subjects actively using recreational THC. The distribution ranged from 0 to $825 \mathrm{ng} / \mathrm{mL}$, with the vast majority of subjects tested at a level of $50 \mathrm{ng} / \mathrm{mL}$ (Figure 6 A). Although impairment measures were not collected in this set of volunteers, this dynamic range study reveals an example expected distribution of THC within an active-intake population.

\subsection{LFI is concordant with DOA MS MRM in a blinded study.}

Due to the rise in marijuana legalization in the United States, police departments have been evaluating rapid THC LFI screening test candidates. Unfortunately, in published studies, THC LFIs can yield unacceptable variability and low sensitivity regardless of the biofluid used. ${ }^{10,23}$ We performed a blinded analysis of $\mathrm{N}=39$ self-reported THC negative patient $\mathrm{OF}$. For all $\mathrm{N}=39$ patients, $100 \%$ returned 
negative by MS analysis (Supplemental Table 2). For a final independent cohort, self-reported THC OF samples were quantified using the MS method and analyzed on the ESS's LFI. The control and test lines of the LFI were quantified via ImageJ densitometry analysis. A digital scan cut-off of 1562 arbitrary units (AU) was used to determine positive (LFI values < cut off) and negative (LFI values > cut off) samples (Figure 6 B). ROC analysis of the patient data led to a LFI sensitivity of $90 \%$ and specificity of $100 \%$ at a threshold of 1562 AU (Figure 6 C). Under object digital scanning the strip, the sensitivity and specificity of the ESS test appears highly accurate under this blinded pilot study confirmed by MS MRM.

We performed a pilot examination of the time course of THC concentration in and perceived drug levels were metabolized quickly to fall well below the legal cut-off of $50 \mathrm{ng} / \mathrm{mL} 1$ hour after smoking, while the levels of perceived impairment remained high (Supplemental Figure 3). Consequently, there is an urgent unmet need to correlate OF parent THC and metabolites with impairment. ${ }^{23}$ In the future conventional impairment scoring can be correlated with OF THC levels using driving simulations.

\section{Conclusions}

348 In conclusion the present study describes an improved protocol for sensitive and accurate MS laboratory confirmation of DOA in oral fluid coupled to a POC device that can both diagnose and store 350 OF simultaneously. Within our patient cohorts, we were able to demonstrate the feasibility and accuracy of the quantitation of the MS test. We used this method to evaluate a novel POC device that collects, diagnoses, and stores a patients unbuffered saliva for DOA screening. The device demonstrated minimal loss of analyte during use, and protects and stores the OF in a stable state for a sufficient time without the need for diluting buffers. The split sample test can be easily deployed and implemented into drug detection programs. Furthermore, the split sample device can be expanded to test up to 52 different analytes and can be customized to rapidly detect and confirm other drugs, antigens, or pathogens of interest, such as COVID-19 within OF. Moreover, alternative pathogen and drug analytes can also be verified by MS in parallel. While the existing pilot study is promising, it has weaknesses in terms of sample number size, and lack of information from participants about level of impairment, or timing since THC use. future studies should include larger patient sets and should evaluate the kinetics of the DOA within OF after drug administration. Additionally, the mode of drug administration should be evaluated, such as inhaled versus ingestion, and correlated with the level of impairment for experienced and first time users. 
365 Among the authors, RG is a shareholder for mLife DX LLC. All other authors affirm that they have no other personal, financial interest, or have received personal income that are related to this topic. The partial-sponsor of this study (mLife DX LLC) had no influence nor role in the content of the

368 analysis of the collection device technology.

\section{Acknowledgments}

370

Oral fluid samples were obtained under patient consent and anonymized following George Mason University's Institutional Review Board approved protocol (No. 1244866-6). Support for this study was provided by the College of Science at George Mason University, the Beck Foundation, and a research grant from mLife DX LLC.

\section{References}

1. Abuse NI on D. National Survey of Drug Use and Health [Internet]. National Institute on Drug Abuse. -- [cited 2020 Sep 11]. Available from: https://www.drugabuse.gov/drug-topics/trendsstatistics/national-drug-early-warning-system-ndews/national-survey-drug-use-health

2. Alderson KG. Addictions Counseling Today: Substances and Addictive Behaviors. SAGE Publications; 2019. $722 \mathrm{p}$. 3. Barrett C, Good C, Moore C. Comparison of point-of-collection screening of drugs of abuse in oral fluid with a laboratory-based urine screen. Forensic Science International. 2001 Nov;122(2-3):1636. 4. Birnbaum HG, White AG, Schiller M, Waldman T, Cleveland JM, Roland CL. Societal Costs of Prescription Opioid Abuse, Dependence, and Misuse in the United States. Pain Med. 2011 Apr;12(4):657-67.

5. Desrosiers NA, Huestis MA. Oral Fluid Drug Testing: Analytical Approaches, Issues and Interpretation of Results. Journal of Analytical Toxicology. 2019 Jul 24;43(6):415-43.

6. Drummer OH. Review: Pharmacokinetics of illicit drugs in oral fluid. Forensic Science International. 2005 Jun 10;150(2):133-42.

7. Gould JB Skye. Legal marijuana just went on sale in Illinois. Here are all the states where cannabis is legal. [Internet]. Business Insider. [cited 2020 Sep 11]. Available from: https://www.businessinsider.com/legal-marijuana-states-2018-1 8. Logan BK, Mohr ALA, Talpins SK. Detection and Prevalence of Drug Use in Arrested Drivers Using the Dräger Drug Test 5000 and Affiniton DrugWipe Oral Fluid Drug Screening Devices. J Anal Toxicol. 2014 Sep 1;38(7):444-50.

9. Malaca S, Busardò FP, Gottardi M, Pichini S, Marchei E. Dilute and shoot ultra-high performance liquid chromatography tandem mass spectrometry (UHPLC-MS/MS) analysis of psychoactive drugs in oral fluid. Journal of Pharmaceutical and Biomedical Analysis. 2019 Jun 5;170:63-7. 10. Morato NM, Pirro V, Fedick PW, Cooks RG. Quantitative Swab Touch Spray Mass Spectrometry for Oral Fluid Drug Testing. Anal Chem. 2019 Jun 4;91(11):7450-7.

402 11. Pehrsson A, Blencowe T, Vimpari K, Langel K, Engblom C, Lillsunde P. An Evaluation of On403 Site Oral Fluid Drug Screening Devices DrugWipe(R) 5+ and Rapid STAT(R) Using Oral Fluid for 404 Confirmation Analysis. Journal of Analytical Toxicology. 2011 May 1;35(4):211-8. 
preparations of medical cannabis and their metabolites in conventional and non-conventional biological matrices of treated individual. Talanta. 2020 Mar;209:120537.

409 13. Reichardt EM, Baldwin D, Osselton MD. Effects of Oral Fluid Contamination on Two Oral Fluid 410 Testing Systems. Journal of Analytical Toxicology. 2013 May 1;37(4):246-9.

411 14. Vandrey R, Herrmann ES, Mitchell JM, Bigelow GE, Flegel R, LoDico C, et al. Pharmacokinetic 412 Profile of Oral Cannabis in Humans: Blood and Oral Fluid Disposition and Relation to

413 Pharmacodynamic Outcomes. J Anal Toxicol. 2017 Mar;41(2):83-99.

414 15. Vereinte Nationen, Büro für Drogenkontrolle und Verbrechensbekämpfung. World drug report 415 2019. 2019.

416 16. Alere iScreen® Dip Card [Internet]. [cited 2020 Sep 10]. Available from:

417 https://www.globalpointofcare.abbott/en/product-details/toxicology-iscreen.html

418 17. Dräger DrugTest ${ }^{\circledR} 5000$ [Internet]. [cited 2020 Sep 10]. Available from:

419 https://www.draeger.com/en_uk/Products/DrugTest-5000

420 18. Driving Under the Influence of Alcohol and Illicit Drugs [Internet]. [cited 2020 Sep 10]. 19. Drug-Impaired Driving: Marijuana and Opioids Raise Critical Issues for States | GHSA [Internet]. [cited 2020 Sep 11]. Available from: https://www.ghsa.org/resources/DUID18 20. Federal Register, Volume 84 Issue 207 (Friday, October 25, 2019) [Internet]. [cited 2019 Dec 17]. Available from: https://www.govinfo.gov/content/pkg/FR-2019-10-25/html/2019-22684.htm 21. Quantisal ${ }^{\mathrm{TM}}$ Oral Fluid Collection Device [Internet]. [cited 2020 Sep 11]. Available from: https://www.globalpointofcare.abbott/en/product-details/quantisal-oral-fluid-collection-deviceau.html 22. SoToxa ${ }^{\mathrm{TM}}$ Mobile Test System [Internet]. [cited 2020 Sep 11]. Available from: https://www.globalpointofcare.abbott/en/product-details/sotoxa-mobile-test-system.html 23. US Patent Application for INTEGRATED DEVICE FOR ANALYTE TESTING, CONFIRMATION, AND DONOR IDENTITY VERIFICATION Patent Application (Application \#20190049442 issued February 14, 2019) - Justia Patents Search [Internet]. [cited 2020 Sep 11]. Available from: https://patents.justia.com/patent/20190049442 24. US Patent for Integrated screening and confirmation device Patent (Patent \# 7,741,103 issued June 22, 2010) - Justia Patents Search [Internet]. [cited 2020 Sep 11]. Available from: https://patents.justia.com/patent/7741103

\section{Figures}


(1)

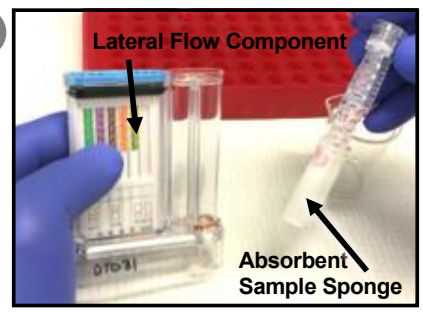

(5)

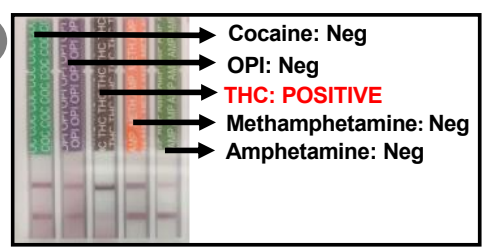

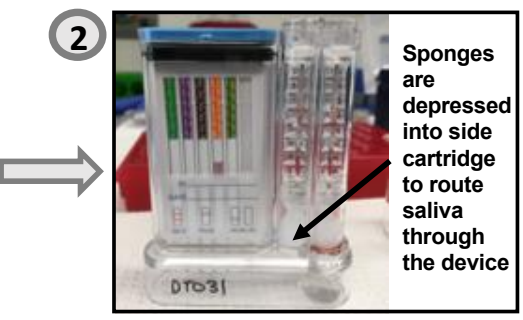
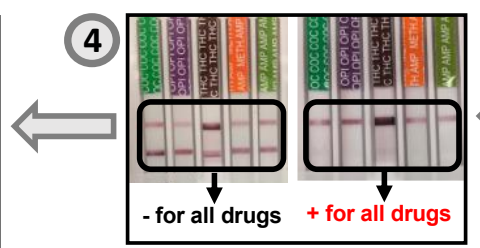
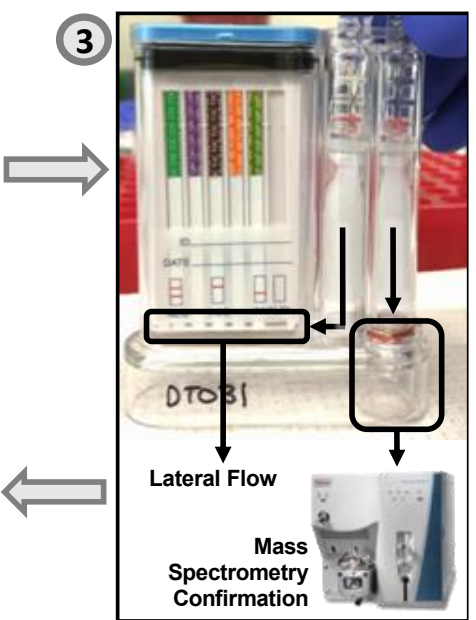

Figure 1. Oral fluid collection system includes dual simultaneous testing and storage for downstream confirmatory analysis. 1) A dual sponge wand is inserted in the mouth and a colorimetric indicator in the wand turns pink when an adequate volume of oral fluid is collected. 2,3) Sponges are depressed and release undiluted oral fluid into two compartments: a) lateral flow immunoassay drug of abuse line and b) secure aliquot for later confirmatory mass spectrometry analysis. 4) The competitive immuno-inhibitory method used in the strips entails 2 visible lines (control at the top and test at the bottom) for negative samples, and 1 visible line (control at the top) for positive samples. 5) Current panel of the multiplex MRM assay includes: cocaine, opioids, THC, 
A)

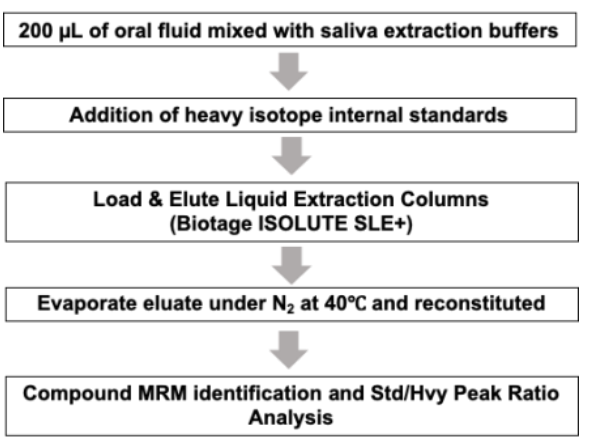

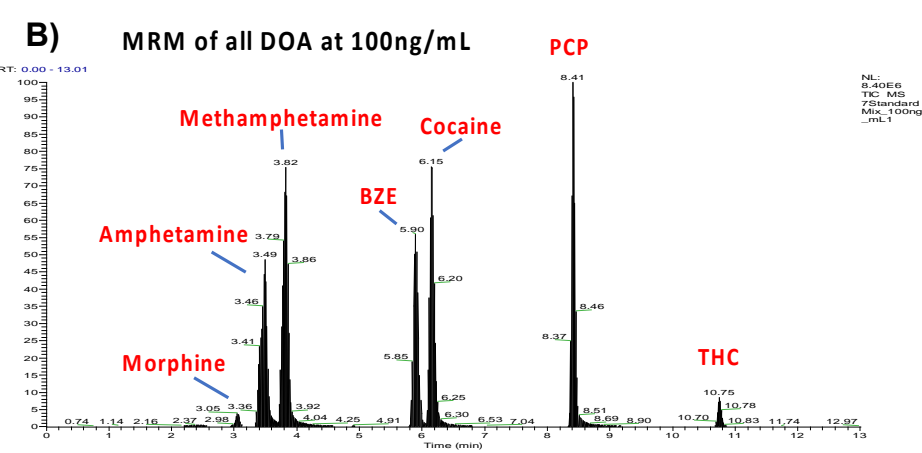
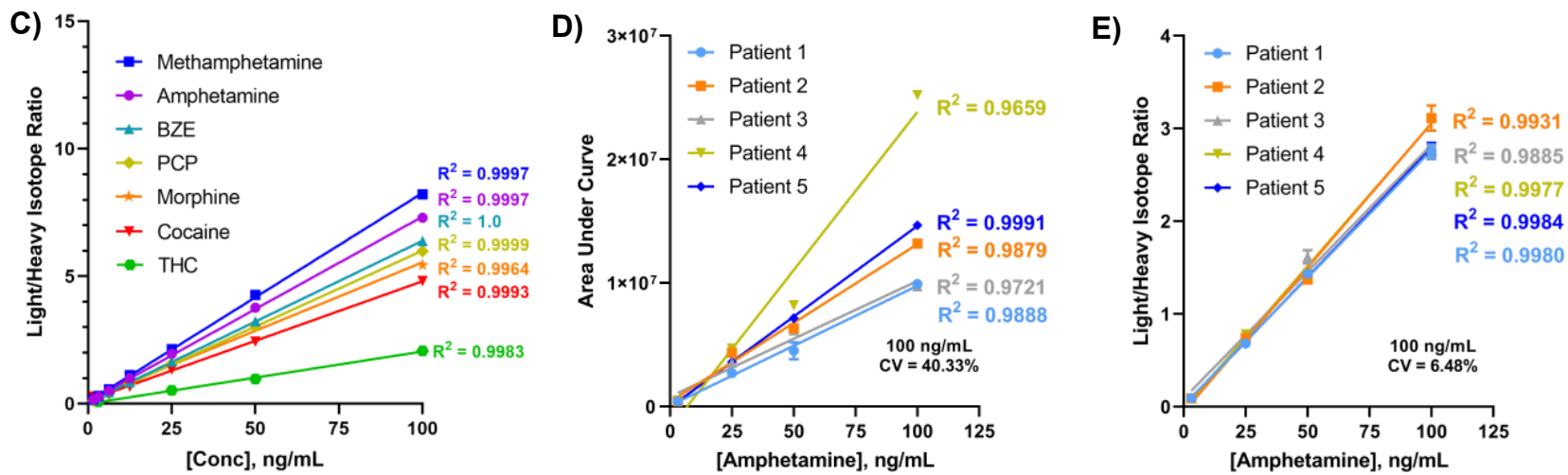

456

457

458

459

460

461

462

463

464

465

466

467

468

469

470

Figure 2. Pre-analytical sample processing and mass spectrometry protocols yield a method that can reproducibly detect seven DOAs in patient oral fluid samples. A) The experimental workflow includes several steps: neat oral fluid is stabilized with buffers and spiked with heavy internal standards prior to sample clean-up via supported liquid extraction columns. Cleaned samples are dried and reconstituted prior to MS MRM analysis. B) MRM chromatogram of 7 DOA at 100 $\mathrm{ng} / \mathrm{mL}$. C) Standard curves for all 7 DOAs in oral fluid show linear regression coefficients $\mathrm{r}^{2}$ higher than 0.999 over a range of $1.56 \mathrm{ng} / \mathrm{mL}$ to $100 \mathrm{ng} / \mathrm{mL}$. D) Peak area quantification of DOA spiked in 5 donors' OF is pronouncedly affected by matrix effects, showing $\% \mathrm{CV}$ of $40.33 \%$ at $100 \mathrm{ng} / \mathrm{mL}$. Data shown for a single drug, Amphetamine. Error bars represent SD for 3 independent measurements. E) Inclusion of a heavy isotope standard and quantification reduces variability due to matrix effects and improves $\mathrm{CV}$ values $(6.48 \%$ at $100 \mathrm{ng} / \mathrm{mL}$ ). For this reason, quantitation was conducted using heavy isotope standards. Data shown for a single drug, Amphetamine. Error bars represent SD for 3 independent measurements. 


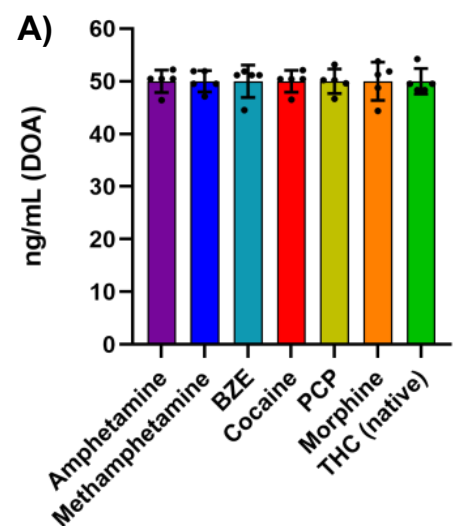

B)

\begin{tabular}{|llll|}
\hline \multicolumn{1}{|c}{ DOA } & Mean & \% CV & N \\
\hline Amphetamine & 50.00 & 4.294 & 5 \\
\hline Methamphetamine & 50.00 & 4.042 & 5 \\
\hline BZE & 50.00 & 6.123 & 5 \\
\hline Cocaine & 50.00 & 4.149 & 5 \\
\hline PCP & 50.00 & 4.623 & 5 \\
\hline Morphine & 50.00 & 7.235 & 5 \\
\hline THC (native) & 50.00 & 4.891 & 5 \\
\hline
\end{tabular}

C)

\begin{tabular}{|l|l|}
\hline \multicolumn{1}{|c|}{ DOA } & LOD $(\mathrm{ng} / \mathrm{mL})$ \\
\hline Amphetamine & $<3.125$ \\
\hline Methamphetamine & $<3.125$ \\
\hline BZE & $<3.125$ \\
\hline Cocaine & $<3.125$ \\
\hline PCP & $<3.125$ \\
\hline Morphine & $<3.125$ \\
\hline THC (native) & $<3.125$ \\
\hline
\end{tabular}

472

473

474

475

476

477

478

479

480

481

482

483

484

485

486

487
D)

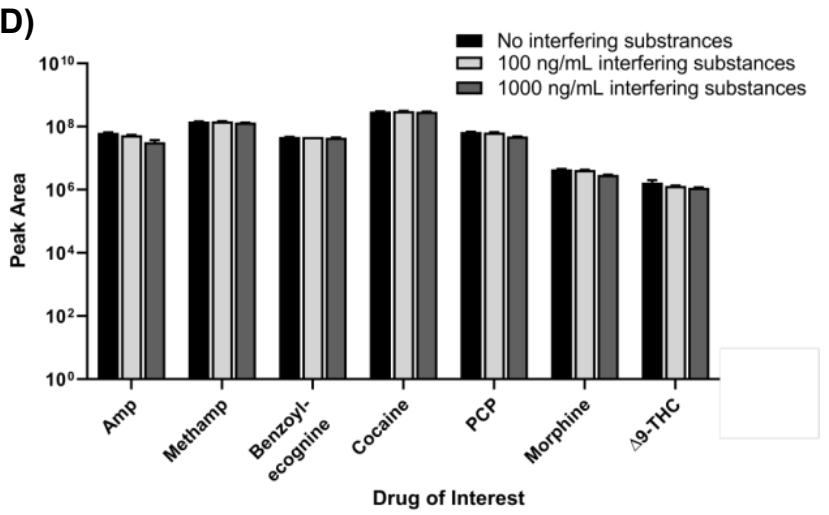

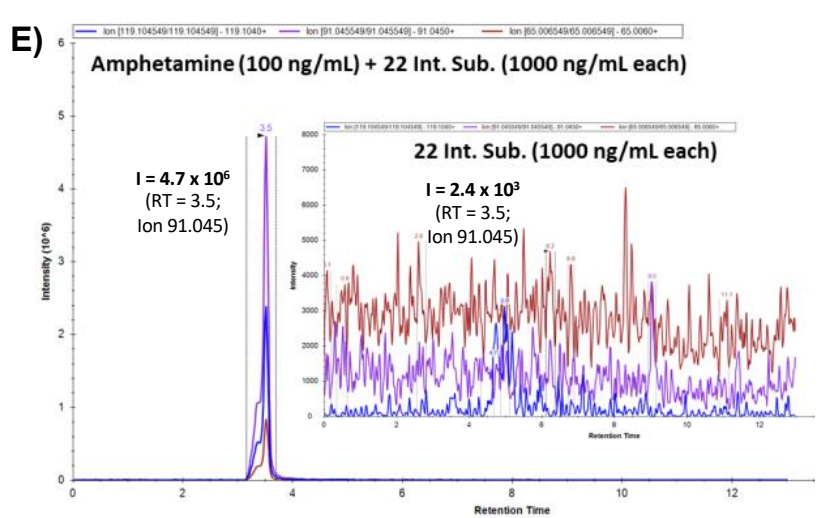

Figure 3. MS MRM detection method for 7 DOA shows high precision, low limits of detection, and is unaffected by the presence of 22 common interfering substances, even at high concentration. A) Individual replicates $(\mathrm{N}=5)$ in a single patient's oral fluid show high reproducibility between MRM analyses. $50 \mathrm{ng} / \mathrm{mL}$ of each drug was spiked into a single patient's oral fluid; individual data point are presented as black dots while error bars represent SD between the 5 independent MRM analyses to demonstrate column extraction efficiency. B) Quantitation of each DOA via std/heavy shows the average of 5 runs for all drugs was $50 \mathrm{ng} / \mathrm{mL}$. All CV values were under 10\%; 5 of the 7 DOAs have CV values under 5\%. C) Limits of detection for each DOA are under $3 \mathrm{ng} / \mathrm{mL}$. D) Peak area was not substantially affected by either 100 or $1000 \mathrm{ng} / \mathrm{mL}$ each of 22 common interfering substances, ensuring effective quantitation. All DOA were spiked at $100 \mathrm{ng} / \mathrm{mL}$. E) Peak intensity of amphetamine fragment ion 91.045 (purple) in the presence of 22 interfering substances is $4.7 \times 10^{6}(\mathrm{RT}=3.5)$. Spectrum of only the 22 interfering substances and no amphetamine shows no signal (only background) at $\mathrm{RT}=3.5$ for fragment ion 91.045 (purple) 

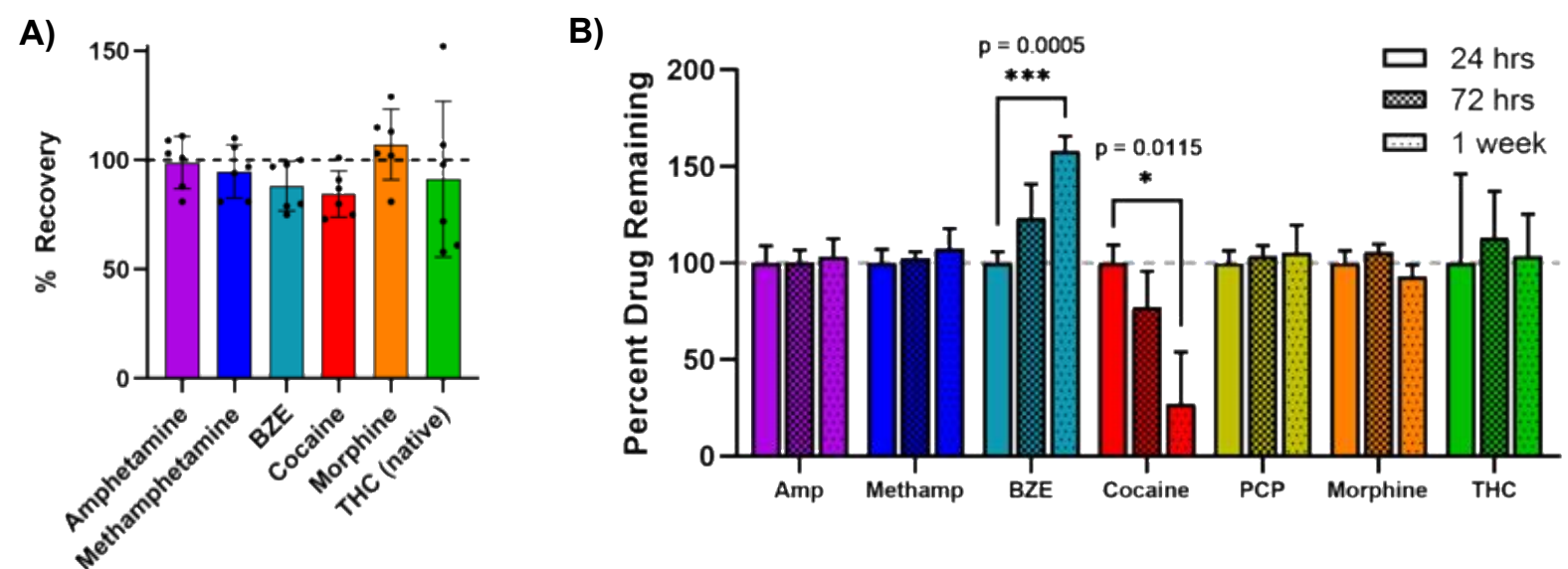

Figure 4. Split sample saliva kits show good recovery of DOAs from the mass spectrometry validation vial, but can be stored at room temperature and analyzed via mass spectrometry no later than 72 hours after collection. A) Recovery of all drugs of abuse from split sample sponge of the collection device was around $100 \%$. $\mathrm{N}=6$ independent experiments. B) Saliva was spiked with 50 $\mathrm{ng} / \mathrm{mL}$ DOAs and quantified at $24 \mathrm{hrs}, 72 \mathrm{hrs}$, and 1 week. Significant degradation was observed only for cocaine; metabolite BZE concentration increased as cocaine decreased, suggesting primary degradation of cocaine was conversion to BZE. Significance was determined via t-test using Graphpad Prism v. 8.0. N = 3 saliva samples per time point from 3 unique individuals. 

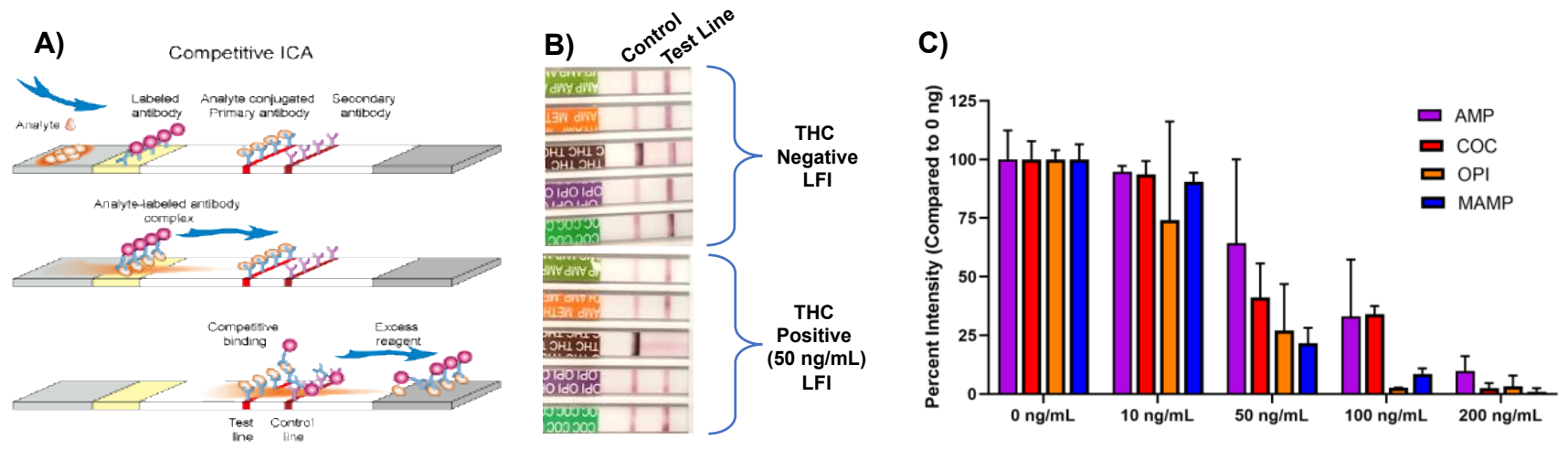

Figure 5. Split-sample oral fluid lateral flow device functions via a competition model and shows dose-dependent LFI response to four DOAs. A) Schematic of lateral flow strips where DOA is detected by labelled antibody. The labelled antibody can competitively bind to either the analyte in the sample (positive, test line does not show up), or to the test line with analyte (negative, test line does show up). B) Example LFI results for both a negative THC sample and a THC-positive sample spiked with $50 \mathrm{ng} / \mathrm{mL}$. C) Percent intensity of the test line for four DOAs was dependent on the dose of DOA in the saliva sample. 
A)

Frequency Distribution $(\mathrm{N}=84)$

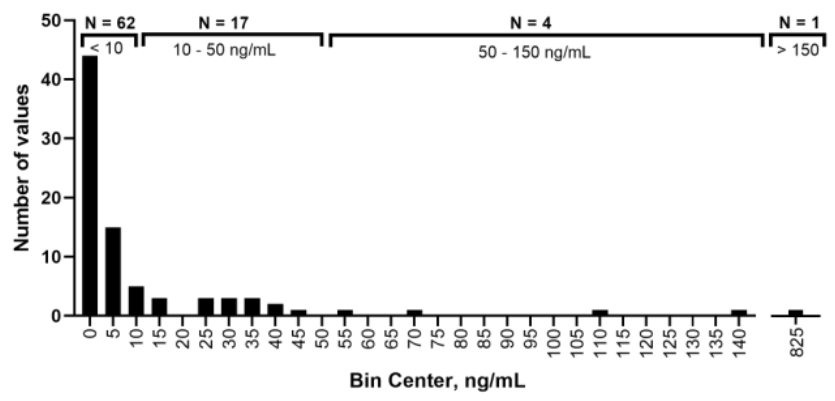

C)

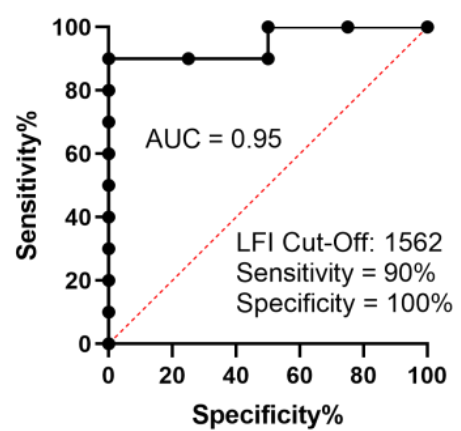

B)

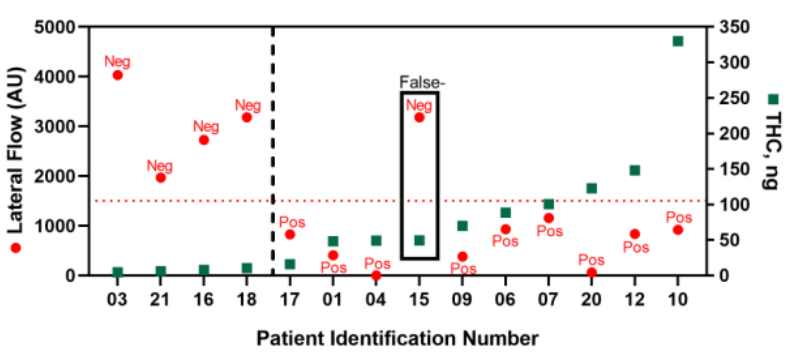

510

511 Figure 6. THC patient cohorts demonstrate wide OF THC dynamic range and split sample

device's THC lateral flows have $93 \%$ concordance with MRM. We examined a cohort of selfreported THC positive OF samples to determine the distribution of THC levels in a given population, and correlate THC patient data to the point of care (POC) diagnosis. A) MRM quantification in a set of recreational THC users revealed a large OF THC dynamic range, from 0-825 ng/mL. B) A blinded MRM study on fourteen independent volunteers who self-reported THC consumption on a single night revealed four negative patients (under $15 \mathrm{ng} / \mathrm{mL} \mathrm{THC),} \mathrm{and} 10$ positive patients (greater than $15 \mathrm{ng} / \mathrm{mL}$ THC). LFI analysis of the same 14 samples revealed 93\% concordance with MRM. C) ROC curve analysis demonstrates that the LFI can determine positive or negative THC status with a sensitivity of $90 \%$ and a specificity of $100 \%$ for 14 patients at a threshold of 1562 arbitrary units. 
A)

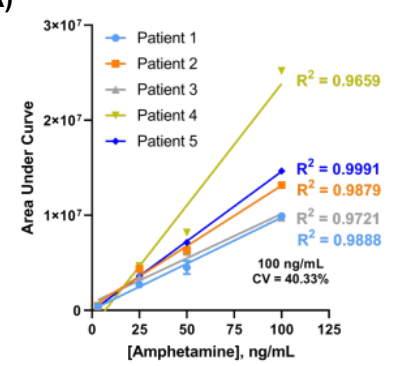

E)

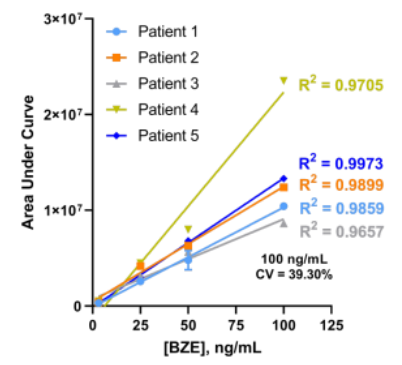

I)

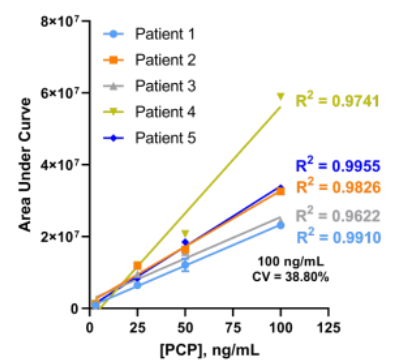

B)

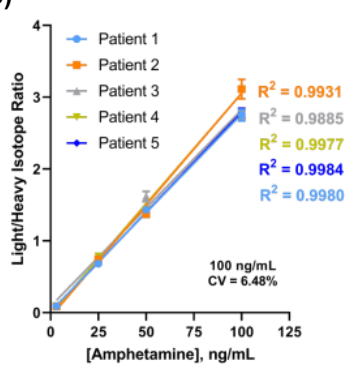

F)

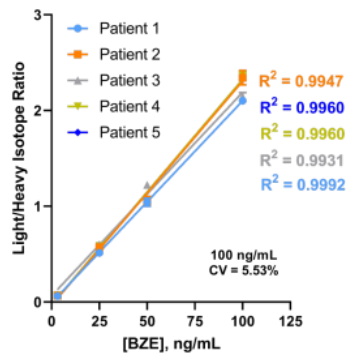

J)

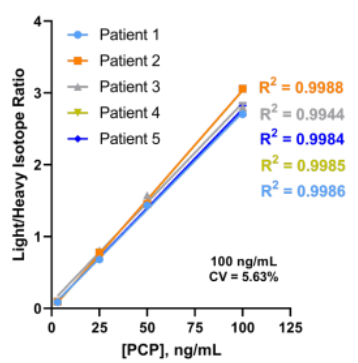

C)

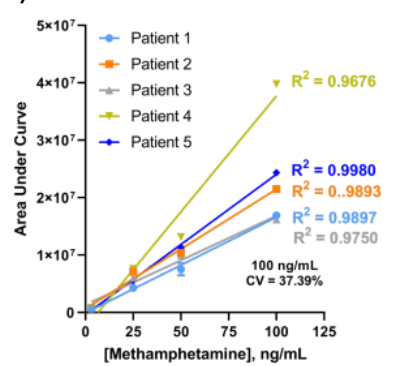

G)

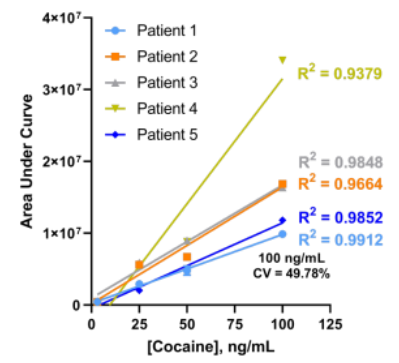

K)

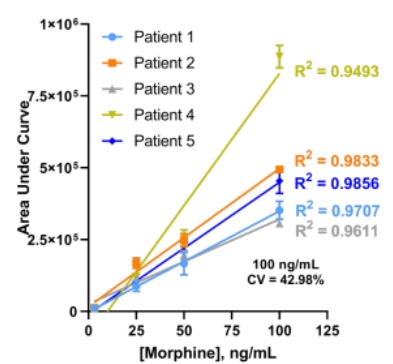

D)

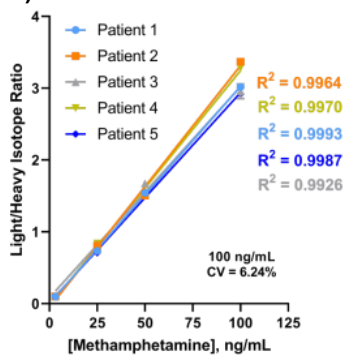

H)

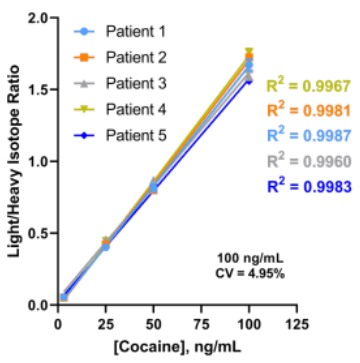

L)

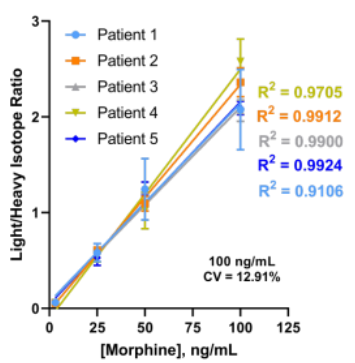

Supplemental Figure 1: Individual DOA area of the curve analysis for quantitation demonstrates higher variability between patients based on matrix effects. The inclusion of standard/heavy ratio $\mathrm{CV}$ values for all drugs analyzed. Error bars represent SD for 3 independent measurements. 
A) Matrix Effects, Methamphetamine (4 days storage)

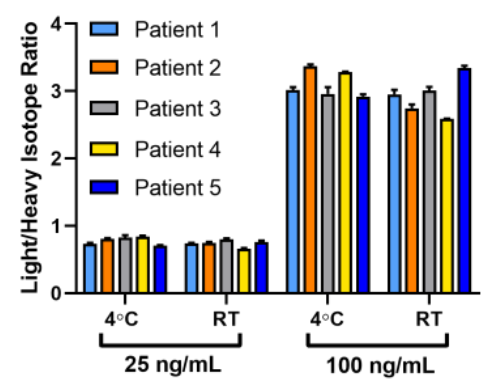

D) Matrix Effects, PCP

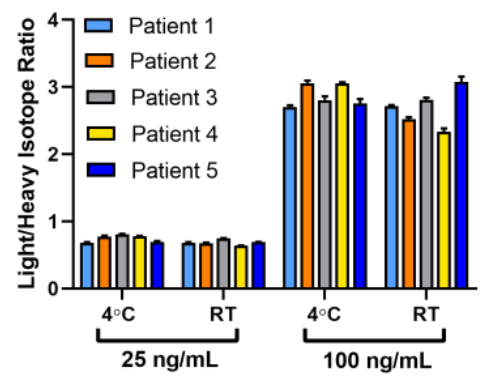

B) Matrix Effects, BZE

(4 days storage)

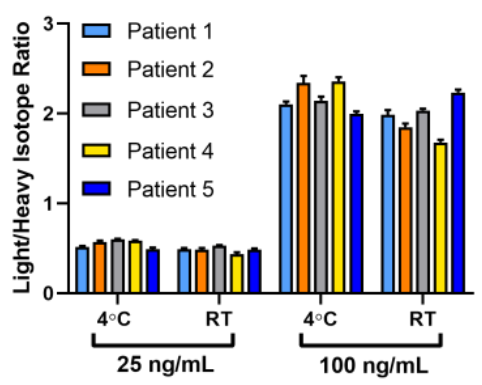

E) Matrix Effects, Morphine

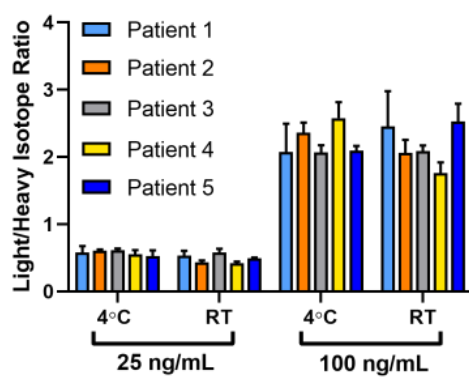

C) Matrix Effects, Cocaine

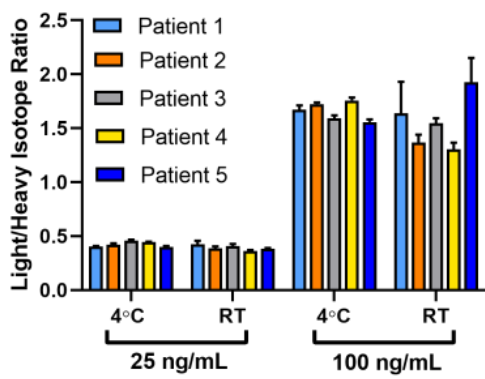

F) Matrix Effects, THC

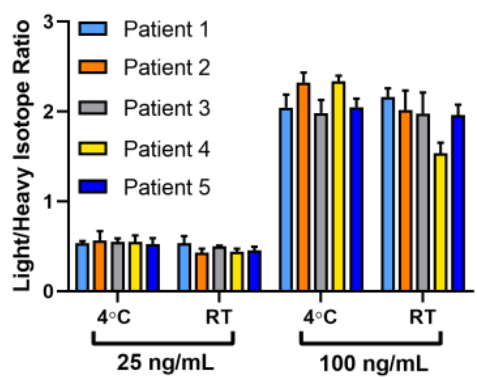

Supplemental Figure 2: Individual DOA stability analysis across N=5 patients at $25 \mathrm{ng} / \mathrm{ml}$ and 100

$529 \mathrm{ng} / \mathrm{mL}$ stored in either $4 \mathrm{C}$ or room temperature over 4 days. Values are the ratio of standard over

530 heavy isotope.

\begin{tabular}{|c|c|c|c|}
\hline Alprazolam & S-Nicotine & Ibuprofen & Verapamil \\
\hline Dihydrocodeine & Caffeine & Naproxen & Sertraline \\
\hline Diphenhydramine & Cortisol & Clonazepam & Prednisone \\
\hline Doxylamine & Cotinine & Valproic Acid & R-Pseudoephedrine \\
\hline Amobarbital & Propranolol & Nicotinamide & Carbamazapine \\
\hline Clobazam & Dextrometho & & \\
\hline
\end{tabular}

531

532

533

534

535

536

537

538

539
Supplemental Table 1: List of Interfering Substances incorporated into the specificity assay in Figure 3.

Supplemental Table 2: Optimal values for MRM determined by direct infusion of all drugs of abuse. Values specified are precursor $\mathrm{m} / \mathrm{z}$, transition $\mathrm{m} / \mathrm{z}$, collision energy (CE) voltage, analysis start and stop time, and tube lens voltage. Transition values shown in bold were used for quantitation.

Supplemental Table 3: Blinded Analysis of N-39 self-reported THC negative unique OF samples via MS returned with $100 \%$ negative results. 


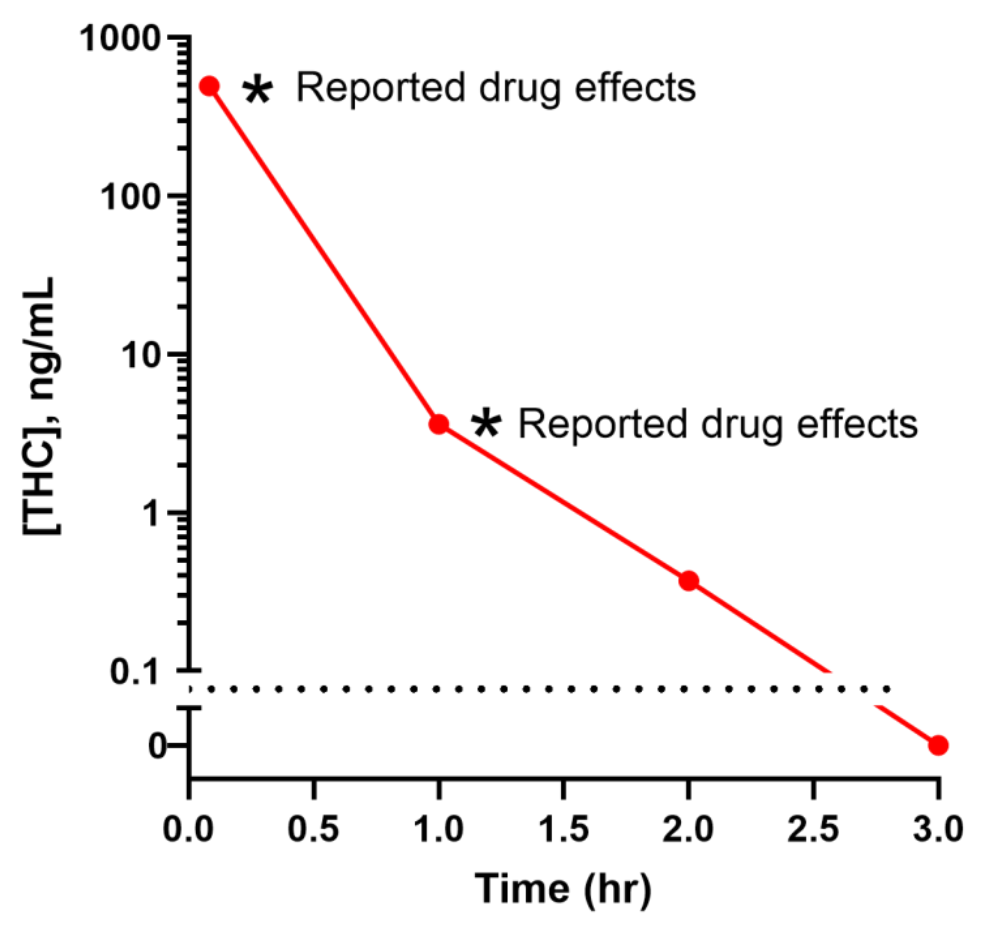

540

541 Supplemental Figure 3: THC time course paired with patient perceived impairment. THC levels 542 were above legal threshold immediately after smoking. At 1-hour post smoking, the individual 543 perceived impairment, however their levels of THC were below the legal cut-off point of $50 \mathrm{ng} / \mathrm{mL}$. 544 Beyond 1 hour, levels of THC continued to decrease linearly until it reached the limit of detection 545 and patient reported no impairment. 